\title{
EXCHANGEABLE RANDOM PARTITIONS FROM MAX-INFINITELY-DIVISIBLE DISTRIBUTIONS
}

\author{
STILIAN STOEV AND YIZAO WANG
}

\begin{abstract}
The hitting partitions are random partitions that arise from the investigation of so-called hitting scenarios of max-infinitely-divisible (maxi.d.) distributions. We study a class of max-i.d. laws with exchangeable hitting partitions obtained by size-biased sampling from the jumps of a Lévy subordinator. We obtain explicit formulae for the distributions of these partitions in the case of the multivariate $\alpha$-logistic and another family of exchangeable max-i.d. distributions. Specifically, the hitting partitions for these two cases are shown to coincide with the well-known Poisson-Dirichlet partitions $\mathrm{PD}(\alpha, 0), \alpha \in(0,1)$ and $\mathrm{PD}(0, \theta), \theta>0$.
\end{abstract}

\section{INTRODUCTION}

Recently there has been a renewed interest in the study of multivariate records in extreme value theory (e.g. 99 11] and references therein), motivated especially by the latest advances on the so-called hitting scenarios for extremal events. The notion of a hitting scenario originated from the investigation of the conditional laws of max-stable processes [4, 25]. In words, a max-stable process can be represented as the pointwise maximum of a family of infinite conditionally independent stochastic processes, and hitting scenarios are introduced to describe whether pointwise maxima at various locations are contributed by a single underlying stochastic process. This notion plays a crucial role in simulation methods for max-stable processes. Hitting scenarios also arise naturally in the expression of the likelihood for max-stable models [20, 23], and in random tessellations determined by max-stable processes [5]. The latest advances on hitting scenarios are motivated by their connections to concurrence probabilities, and the framework can be naturally stated in the language of random partitions [6, 7].

Our focus here is on the probabilistic aspects of the hitting partitions of multivariate max-stable distributions, recently introduced in [6]. A hitting partition can be viewed, more generally, as a random partition derived from the hitting scenario of a max-stable process $[3,24]$. We focus however on finite-dimensional distributions most of the time for the sake of simplicity. We begin with recalling the definition. First, let $\left\{\xi_{\ell}\right\}_{\ell \in \mathbb{N}}$ be a measurable enumeration of points of a Poisson point process on $\mathbb{R}_{+}$with intensity $\nu_{\alpha}(d x):=\alpha x^{-\alpha-1} d x$ for some $\alpha>0$, and let $\left\{\boldsymbol{Y}_{\ell}\right\}_{\ell \in \mathbb{N}}$ be i.i.d. copies of certain non-negative random vector $\boldsymbol{Y}=\left(Y_{1}, \ldots, Y_{n}\right)$ with finite $\alpha$-moments. Throughout, $\mathbb{N}=\{1,2, \ldots\}$ and $\mathbb{N}_{0}=\mathbb{N} \cup\{0\}$. Then, it is well known

Date: September 5, 2021.

2010 Mathematics Subject Classification. Primary, 60G70, Secondary, 60C05.

Key words and phrases. exchangeable random partition, multivariate max-infinitely-divisible distribution, Poisson-Dirichlet distribution, paintbox partition. 
that the vector

$$
\boldsymbol{Z} \equiv\left(Z_{1}, \ldots, Z_{n}\right) \equiv\left(\bigvee_{\ell=1}^{\infty} \xi_{\ell} Y_{\ell, 1}, \ldots, \bigvee_{\ell=1}^{\infty} \xi_{\ell} Y_{\ell, n}\right)
$$

has a multivariate max-stable $\alpha$-Fréchet distribution [3]. Throughout, we write $\vee \equiv$ max. Recall that a random variable $Z$ is said to be $\alpha$-Fréchet if

$$
\mathbb{P}(Z \leq x)=\exp \left(-\sigma^{\alpha} / x^{\alpha}\right), \quad \text { for } x \in(0, \infty),
$$

with scale parameter $\sigma>0$. The vector $\boldsymbol{Z}$ is max-stable with $\alpha$-Fréchet marginals if all its non-negative max-linear combinations $\bigvee_{k=1}^{n} a_{k} Z_{k}$ are $\alpha$-Fréchet distributed, for all $a_{k} \geq 0, k=1, \ldots, n$. The representation (1.1) is an instance of the so-called Le Page-type series representations in the special case of the semi-group $\left(\mathbb{R}^{d}, \vee\right)[2]$.

Given a max-stable Fréchet random vector with the representation (1.1), the induced hitting partition is determined as follows. Set

$$
\ell^{*}(k):=\operatorname{argmax}_{\ell \in \mathbb{N}}\left\{\xi_{\ell} Y_{\ell, k}\right\}, k=1, \ldots, n .
$$

Note that $\left\{\xi_{\ell} Y_{\ell, k}, \ell \in \mathbb{N}\right\}$ is a simple Poisson process on $(0, \infty)$ and hence with probability one, $\ell^{*}(k)$ is uniquely determined for every $k$; we restrict ourselves to this event from now on.

The hitting partition of $[n] \equiv\{1, \ldots, n\}$, for $n \in \mathbb{N}$, denoted by $\Pi_{n}$, is the random partition of equivalence classes induced by the relation

$$
i \sim j \quad \text { if and only if } \quad \ell^{*}(i)=\ell^{*}(j), \text { for all } i, j \in[n] .
$$

Here, $i \sim j$ reads as $i$ and $j$ are in the same block of the partition. Recall that a partition of $[n]$ is a collection of disjoint sets, the union of which is $[n]$.

Thus far, most of the research on the hitting partitions has focused on the socalled concurrence probability, that is, the probability of the event that the hitting partition $\Pi_{n}$ consists of a single block [6, 7]. The concurrence probability has the following expression

$$
p(n) \equiv \mathbb{P}\left(\Pi_{n}=\{[n]\}\right)=\mathbb{E}\left(\frac{1}{\mathbb{E}\left(\bigvee_{k=1}^{n}\left(Y_{k}^{*} / Y_{k}\right)^{\alpha} \mid \boldsymbol{Y}\right)}\right),
$$

where $\boldsymbol{Y}^{*}$ is an independent copy of $\boldsymbol{Y}$. This result was established in $\underline{6}$, Theorem 2 ] by using the Slyvniak-Mecke formula, and the same method in principle could yield formulae for the entire probability distribution of the hitting partition (see, e.g. [4]). The general expressions are however neither explicit nor intuitive.

The motivation of this paper is to study specific choices of $\boldsymbol{Y}$, where the induced hitting partition has an explicit probability mass function. Our starting point is an example from a very recent paper [6, Example 3], where $\alpha \in(0,1)$ and $\boldsymbol{Y}=\left(Y_{1}, \ldots, Y_{n}\right)$ has i.i.d. 1-Fréchet components. Then, the distribution of $\boldsymbol{Z}=$ $\left(Z_{1}, \ldots, Z_{n}\right)$ in (1.1) becomes multivariate $\alpha$-logistic (see Example 3.4 below). In this case, the concurrence probability has a simple-looking formula

$$
p(n)=\prod_{k=1}^{n-1}\left(1-\frac{\alpha}{k}\right) \equiv \frac{\prod_{k=1}^{n-1}(k-\alpha)}{(n-1) !}, n \in \mathbb{N} .
$$

In this paper, first we explain this formula by showing that the hitting partition in this case is the exchangeable random partition induced by the Poisson-Dirichlet distribution with parameters $(\alpha, 0)$. Poisson-Dirichlet distributions and exchangeable random partitions are fundamental objects in combinatorial stochastic processes, 
with numerous applications, notably in nonparametric inference and population genetics [1, 17]. An outstanding family of exchangeable random partitions are the ones induced by the Poisson-Dirichlet distribution with parameters $\alpha, \theta$, referred to as the $\mathrm{PD}(\alpha, \theta)$ partitions for short. The legitimate values of the parameters are $\alpha<0, \theta=-m \alpha$ for some $m \in \mathbb{N}$ or $\alpha \in[0,1], \theta>-\alpha$. For any selected partition of $[n]$ with block sizes $n_{1}, \ldots, n_{k}$ (such that $n_{1}+\cdots+n_{k}=n, n_{1}, \ldots, n_{k} \geq 1$ ), the probability of a $\operatorname{PD}(\alpha, \theta)$ taking the value of this partition equals

$$
p_{\alpha, \theta}\left(n_{1}, \ldots, n_{k}\right)=\frac{(\theta+\alpha)_{k-1 \uparrow \alpha} \prod_{i=1}^{k}(1-\alpha)_{n_{i}-1 \uparrow 1}}{(\theta+1)_{n-1 \uparrow \alpha}},
$$

where $(x)_{m \uparrow \alpha}:=\prod_{k=0}^{m-1}(x+k \alpha)$. (See [17, Theorem 3.2, Definition 3.3].) The Poisson-Dirichlet random partitions are actually exchangeable random partitions of $\mathbb{N}$, although we focus on their restriction to $[n](1.4)$ most of the time.

Proposition 1.1. The hitting partition $\Pi_{n}$ of the $\alpha$-logistic max-stable model, $\alpha \in$ $(0,1)$, is a $\mathrm{PD}(\alpha, 0)$ partition.

The result follows essentially from the paintbox representation of exchangeable random partitions, to be reviewed in Section 2, and the max-stability property of Fréchet distributions. By recognizing the random weights in the paintbox representation as the (normalized) jumps of an $\alpha$-stable subordinator, we obtain that the hitting partition is in fact the $\operatorname{PD}(\alpha, 0)$ partition. Thus, our method is completely different from the one applied in [6].

Moreover, it turns out that the same idea of the proof can be applied to a larger family of hitting partitions, associated with a class of max-infinitely-divisible (maxi.d.) distributions [18]. The latter are obtained as in (1.1), but by considering a general intensity measure $\nu$ for the Poisson process $\left\{\xi_{\ell}, \ell \in \mathbb{N}\right\}$ while keeping the independent $\alpha$-Fréchet marks $Y_{\ell, k}$ 's. The resulting max-i.d. distributions will be referred to as sub-Fréchet max-i.d. distributions.

Our main result is Theorem 2.3. which establishes a paintbox representation of the hitting partitions for all sub-Fréchet max-i.d. distributions: these hitting partitions are precisely the exchangeable random partitions obtained via size-biased sampling of jumps from a subordinator with Lévy measure $\nu$ [17, Chapter 4.1]. This representation allows us to identify another class of non-max-stable sub-Fréchet max-i.d. distributions, whose hitting partitions have the $\operatorname{PD}(0, \theta)$ laws, for $\theta>0$.

The paper is organized as follows. In Section 2 we review the background on exchangeable random partitions and prove the main result Theorem 2.3. In Section 3 we provide related results on the sub-Fréchet max-i.d. distributions.

\section{Hitting PARTitions of SUB-FrÉCHET MAX-I.D. DistribUtions}

We shall consider a multivariate max-i.d. distribution with the following representation

$$
\left(\zeta_{1}, \ldots, \zeta_{n}\right) \equiv\left(\bigvee_{\ell=1}^{\infty} J_{\ell} Y_{\ell, 1}, \ldots, \bigvee_{\ell=1}^{\infty} J_{\ell} Y_{\ell, n}\right),
$$

where $\boldsymbol{J} \equiv\left\{J_{\ell}\right\}_{\ell \in \mathbb{N}}$ is a Poisson point process on $\mathbb{R}_{+}$with intensity measure $\nu$, and $\left\{\boldsymbol{Y}_{\ell}\right\}_{\ell \in \mathbb{N}}$ are i.i.d. random vectors independent from $\boldsymbol{J}$, each $\boldsymbol{Y}_{\ell}=\left(Y_{\ell, 1}, \ldots, Y_{\ell, n}\right)$ is a collection of independent 1-Fréchet random variables, with scale parameters 
$\boldsymbol{\sigma}=\left(\sigma_{1}, \ldots, \sigma_{n}\right) \in(0, \infty)^{n}$. The values of $\boldsymbol{\sigma}$ shall not have any impact in most of the discussions until Section 3 ,

We assume throughout that

$$
\nu\left(\mathbb{R}_{+}\right)=\infty \quad \text { and } \quad \int_{0}^{\infty}(1 \wedge x) \nu(d x)<\infty .
$$

This ensures that the Poisson process $\boldsymbol{J}$ has infinitely many points, and

$$
J_{*}:=\sum_{\ell=1}^{\infty} J_{\ell}<\infty \text { a.s. }
$$

In particular, the random variables the $\zeta_{i}$ 's in (2.1) are finite, almost surely. This readily follows from the max-stability property of the $Y_{\ell, k}$ 's. Namely, by the fact that, for all $a_{\ell} \geq 0, \ell \in \mathbb{N}$ with $\sum_{\ell=1}^{\infty} a_{\ell}<\infty$, we have

$$
\bigvee_{\ell=1}^{\infty} a_{\ell} Y_{\ell} \stackrel{d}{=}\left(\sum_{\ell=1}^{\infty} a_{\ell}\right) Y_{1}
$$

for i.i.d. 1-Fréchet random variables $\left\{Y_{\ell}\right\}_{\ell \in \mathbb{N}}$.

We name the max-i.d. random vector $\zeta=\left(\zeta_{1}, \ldots, \zeta_{n}\right)$ in (2.1), as a sub-Fréchet max-i.d. random vector with Lévy measure $\nu$. The terminology is inspired by the corresponding sub-stable distributions [19] (see (3.3) below).

Definition 2.1. For all $n \in \mathbb{N}$, set

$$
\ell^{*}(k):=\operatorname{argmax}_{\ell \in \mathbb{N}}\left\{J_{\ell} Y_{\ell, k}\right\}, k=1, \ldots, n,
$$

and define now the random partition $\Pi_{n}$ of $[n]$ for $n \in \mathbb{N}$ by (1.2) as before. We refer to the so-defined random partition as the hitting partition of the maxi.d. distribution in (2.1).

As before, $\ell^{*}(k)$ in (2.3) is uniquely defined with probability one. Indeed, for every pair $\ell, \ell^{\prime} \in \mathbb{N}, \ell \neq \ell^{\prime}$, it is easy to see that with probability one $J_{\ell} Y_{\ell, k} \neq J_{\ell^{\prime}} Y_{\ell^{\prime}, k}$ by conditioning on the values of $J_{\ell}, J_{\ell^{\prime}}$, as the law of $Y_{\ell, k}$ has no atom.

Remark 2.2. Following [18, Chapter 5], given a non-negative max-i.d. vector, say $\boldsymbol{\zeta}=\left(\zeta_{1}, \ldots, \zeta_{n}\right)$, there exists a unique measure $\mu$ on $\left(\mathbb{R}_{+}^{n}, \mathcal{B}\left(\mathbb{R}_{+}^{n}\right)\right)$, known as the Lévy (or exponent) measure of $\boldsymbol{\zeta}$, such that for all $\boldsymbol{x}=\left(x_{1}, \ldots, x_{n}\right) \in \mathbb{R}_{+}^{n}$,

$$
\mathbb{P}\left(\zeta_{k} \leq x_{k}, k=1, \ldots, n\right)=\exp \left(-\mu\left([\mathbf{0}, \boldsymbol{x}]^{c}\right)\right) .
$$

Thus, taking a Poisson point process $\boldsymbol{\Psi}=\left\{\boldsymbol{\Psi}_{\ell}\right\}_{\ell \in \mathbb{N}}$ on $\mathbb{R}_{+}^{n}$ with mean measure $\mu$, we obtain the stochastic representation

$$
\left(\zeta_{1}, \ldots, \zeta_{n}\right) \stackrel{d}{=}\left(\sup _{\ell \in \mathbb{N}} \Psi_{\ell, 1}, \ldots, \sup _{\ell \in \mathbb{N}} \Psi_{\ell, n}\right) .
$$

The law of the hitting partition depends only on the law of $\boldsymbol{\Psi}$, which in turn is uniquely determined by the law of $\boldsymbol{\zeta}$ as argued in [6]. However, our starting point (2.1) is the assumption that the Poisson point process $\boldsymbol{\Psi}$ has the following specific representation

$$
\left\{\boldsymbol{\Psi}_{\ell}\right\}_{\ell \in \mathbb{N}} \stackrel{d}{=}\left\{J_{\ell} \boldsymbol{Y}_{\ell}\right\}_{\ell \in \mathbb{N}} .
$$

Many different spectral representations of this form are possible. Here, we assume that one exists where the random vector $\boldsymbol{Y}$ has independent 1-Fréchet components, which plays a crucial role in deriving formulae of random partitions. See Remark 3.2 for an alternative characterization of the exponent measure $\mu$ in this case. 
Our main result is to show that in this framework, the random partition $\Pi_{n}$ is exchangeable, and it has the same law as a paintbox partition (a.k.a. partition generated by random sampling) with random weights

$$
P_{\ell}:=\frac{J_{\ell}}{\sum_{\ell^{\prime}=1}^{\infty} J_{\ell^{\prime}}}, \ell \in \mathbb{N} \quad \text { and } \quad P_{0}:=0 .
$$

We first review the background of the paintbox construction 1, 17]. Recall that a paintbox partition with respect to weight $s=\left(s_{0}, s_{1}, \ldots\right)$ such that $s_{0} \geq 0, s_{1} \geq$ $s_{2} \geq \cdots \geq s_{n} \geq \cdots \geq 0$ and $\sum_{k=0}^{\infty} s_{k}=1$, is a canonical way to obtain exchangeable random partitions of $\mathbb{N}$ as follows. Let $\left\{X_{n}\right\}_{n \in \mathbb{N}}$ be i.i.d. sampling from $\mathbb{N}_{0}$ with distribution $\mathbb{P}\left(X_{1}=\ell\right)=s_{\ell}, \ell \in \mathbb{N}_{0}$. Color the set of natural numbers $\mathbb{N}=$ $\{1,2, \ldots\}$ as follows. If $X_{i}>0$, we paint $i$ in color $X_{i}$, otherwise, all $i$ 's with $X_{i}=0$ are colored in different colors that are also different from all other colors used in the paintbox. Thus $\mathbb{N}$ is partitioned into disjoint blocks by different colors. Formally, this partition is induced by the equivalence relation $i \sim j$ if $X_{i}=X_{j}>0$ for all $i, j \in \mathbb{N}$. Notice that every $i \in \mathbb{N}$ such that $X_{i}=0$ forms a singleton block by itself.

It is well known and easy to see that the so-obtained partition of $\mathbb{N}$ is exchangeable. Moreover, Kingman's representation theorem [17, Theorem 2.2] says that every exchangeable random partition of $\mathbb{N}$ can be obtained by a paintbox partition with possibly random weights $s$. In this case, conditionally on $s,\left\{X_{n}\right\}_{n \in \mathbb{N}}$ are i.i.d. with distribution $\mathbb{P}\left(X_{1}=\ell \mid s\right)=s_{\ell}, \ell \in \mathbb{N}_{0}$.

Therefore, a convenient way to characterize the law of an infinite exchangeable partition is to identify the random weights of the corresponding paintbox partition. Our discussions focus on finite partitions: if a finite partition can be obtained by a paintbox partition with a finite number of i.i.d. samplings, it is clearly exchangeable, with the law determined by the weights, and we still refer to it as a paintbox partition.

Theorem 2.3. For all $n \in \mathbb{N}$, the hitting partition $\Pi_{n}$ associated to (2.1) is an exchangeable random partition of $[n]$, which has the same law as a paintbox partition with random weights $\left\{P_{\ell}\right\}_{\ell \in \mathbb{N}_{0}}$ given by (2.5).

Proof. To see this, we first observe that conditioning on $\boldsymbol{J}$, for each $k=1, \ldots, n$, the distribution of $\ell^{*}(k)$ is determined by $\mathbb{P}\left(\ell^{*}(k)=\ell \mid \boldsymbol{J}\right), \ell \in \mathbb{N}$, and conditionally on $\boldsymbol{J}$, we have that $\left\{\ell^{*}(k)\right\}_{k=1, \ldots, n}$ are independent, since so are the $Y_{\ell, k}$ 's. The probability of interest turns out to be independent from $k$. Indeed, we have

$$
\begin{aligned}
\mathbb{P}\left(\ell^{*}(k)=\ell \mid \boldsymbol{J}\right) & =\mathbb{P}\left(J_{\ell} Y_{\ell, k}>\max _{\ell^{\prime} \neq \ell} J_{\ell^{\prime}} Y_{\ell^{\prime}, k} \mid \boldsymbol{J}\right) \\
& =\mathbb{P}\left(J_{\ell} Y_{1, k}>\left(\sum_{\ell^{\prime} \neq \ell} J_{\ell^{\prime}}\right) Y_{2, k} \mid \boldsymbol{J}\right) \\
& =\frac{J_{\ell}}{\sum_{\ell^{\prime}=1}^{\infty} J_{\ell^{\prime}}}=P_{\ell}, \ell \in \mathbb{N} .
\end{aligned}
$$

Relation (2.6) follows from the max-stability property (2.2) of the Fréchet distribution, while Relation (2.7) follows from the property

$$
\mathbb{P}\left(a Y_{1}>b Y_{2}\right)=\frac{a}{a+b},
$$


valid for all $a, b>0$ where $Y_{1}$ and $Y_{2}$ are i.i.d. 1-Fréchet random variables. This completes the proof.

The aforementioned framework of exchangeable random partitions based on jump sizes of subordinators, via (2.5), is well known. In fact, if the Lévy measure $\nu$ has a density $\rho$, then under mild conditions explicit formula for the random partition generalizing (1.4) is available in terms of $\rho$. See [16] and [17, Exercise 4.1.2]. To keep the presentation short, we shall instead explain only two special examples in full detail here.

Recall that the Poisson-Dirichlet distribution refers to a two-parameter family of ranked frequencies of $\left\{P_{\ell}\right\}_{\ell \in \mathbb{N}}$, indexed by $(\alpha, \theta)$ with either $\alpha<0, \theta=-m \alpha$ for some $m \in \mathbb{N}$, or $\alpha \in[0,1], \theta>-\alpha$. When the frequencies are ordered in sizebiased order, the corresponding law of the same two-parameter family is known as the Griffiths-Engen-McCloskey (GEM) distribution. The size-biased frequencies, denoted by $\left\{\widetilde{P}_{\ell}\right\}_{\ell \in \mathbb{N}}$, have the representation

$$
\left(\widetilde{P}_{1}, \widetilde{P}_{2}, \widetilde{P}_{3}, \ldots\right) \stackrel{d}{=}\left(W_{1},\left(1-W_{1}\right) W_{2},\left(1-W_{1}\right)\left(1-W_{2}\right) W_{3}, \ldots\right),
$$

where $\left\{W_{\ell}\right\}_{\ell \in \mathbb{N}}$ are independent beta random variables, each with parameters (1$\alpha, \theta+\ell \alpha)$. Here, formally the size-biased frequencies are defined iteratively as follows: given the probability on $\mathbb{N}$ determined by $\left\{P_{\ell}\right\}_{\ell \in \mathbb{N}}$, consider a sequence of i.i.d. sampling from this probability, and let $\widetilde{P}_{1}$ denote the probability of the first label sampled, $\widetilde{P}_{2}$ denote the second new label sampled, and so on.

In particular, explicit examples relating random weights from subordinators via (2.5) to Poisson-Dirichlet distributions have been well known, and we shall make use of the following two from them (see [17, Chapter 4.2]):

(i) If $\nu(d x)=\alpha x^{-\alpha-1} d x, \alpha \in(0,1)$, then the subordinator is an $\alpha$-stable subordinator. The ranked frequencies have the law of $\operatorname{PD}(\alpha, 0)$.

(ii) If $\nu(d x)=\theta x^{-1} e^{-x} d x, \theta>0$, then the subordinator is a Gamma process. The ranked frequencies have the law of $\operatorname{PD}(0, \theta)$.

The next corollary follows immediately, including Proposition 1.1 as the first case.

Corollary 2.4. For the sub-Fréchet max-i.d. distribution (2.1) with Lévy measure $\nu$, the induced hitting partition $\Pi_{n}$ is:

(i) $\mathrm{PD}(\alpha, 0), \alpha \in(0,1)$ if $\nu(d w)=\alpha w^{-\alpha-1} d w$.

(ii) $\mathrm{PD}(0, \theta), \theta>0$ if $\nu(d w)=\theta w^{-1} e^{-w} d w$.

Remark 2.5. The paintbox argument in the proof of Theorem 2.3 applies without change to the case where (2.1) is an infinite max-i.d. sequence indexed by $\mathbb{N}$. In this case, one obtains exchangeable partitions of $\mathbb{N}$. We stated Theorem 2.3 in the finite-dimensional setting of partitions on $[n]$ for simplicity and in order to draw connections to the exiting results on the concurrence probability (e.g. formula (1.3) ).

Remark 2.6. The class of hitting partitions arising from (2.1) does not contain all exchangeable random partitions. By allowing dependence among, and/or other types of distributions of, $Y_{\ell, 1}, \ldots, Y_{\ell, n}$, one could obtain other exchangeable random partitions by the same mechanism. In particular, it seems that $\operatorname{PD}(\alpha, \theta)$ partitions for other choices of the parameters do not appear as the hitting partitions of subFréchet max-i.d. laws. 


\section{Distributions of SUB-FrÉChet MAX-I.D. Distributions}

So far, we have introduced a specific family of max-i.d. distributions and shown that the induced hitting partitions have more explicit structure. In this section, we collect some facts on this family of max-i.d. distributions. All the computations are straightforward and standard, and are hence omitted.

Proposition 3.1. For $\left(\zeta_{1}, \ldots, \zeta_{n}\right)$ as in (2.1),

$$
\mathbb{P}\left(\zeta_{k} \leq x_{k}, k=1, \ldots, n\right)=L_{J_{*}}\left(\sum_{k=1}^{n} \frac{\sigma_{k}}{x_{k}}\right), x_{k} \geq 0, k=1, \ldots, n,
$$

where

$$
L_{J_{*}}(t):=\mathbb{E} \exp \left(-t J_{*}\right)=\exp \left(-\int_{0}^{\infty}\left(1-e^{-t x}\right) \nu(d x)\right), t>0
$$

is the Laplace transform of $J_{*}$. In particular,

$$
\left(\zeta_{1}, \ldots, \zeta_{n}\right) \stackrel{d}{=} J_{*}\left(Y_{1,1}, \ldots, Y_{1, n}\right) .
$$

Remark 3.2. Relations (3.1) and (2.4) imply that a positive max-i.d. random vector $\zeta$ is sub-Fréchet with exponent measure $\nu$ as in (2.1), if and only if

$$
\mu\left([\mathbf{0}, \boldsymbol{x}]^{c}\right)=g_{\nu}\left(\sum_{k=1}^{n} \frac{\sigma_{k}}{x_{k}}\right), \boldsymbol{x} \in(0, \infty)^{n},
$$

where $g_{\nu}(t) \equiv-\log L_{J^{*}}(t)=\int_{0}^{\infty}\left(1-e^{-t x}\right) \nu(d x), t \geq 0$ is the Laplace exponent of $J^{*}$ as in (3.2) and $\boldsymbol{\sigma} \in(0, \infty)^{n}$. Note that $g_{\nu}$ is a Bernstein function in general.

Remark 3.3. A priori, it is not obvious that for what functions $L_{J_{*}}$ the right-hand side of (3.1) defines a valid multivariate distribution function. An alternative proof of this fact can be obtained from the perspective of Archimedean copula [8, 13]. The recent work of [12] uses related ideas on the elegant properties of strongly sum infinitely divisible laws to study a class of multivariate max-stable laws (formulated as extreme-value copula).

Example 3.4. For the two cases of Corollary 2.4, we have accordingly the following explicit formulae.

(i) $L_{J_{*}}(t)=e^{-t^{\alpha}}$, and reads as

$$
\mathbb{P}\left(\zeta_{k} \leq x_{k}, k=1, \ldots, n\right)=\exp \left(-\left(\sum_{k=1}^{n} \frac{\sigma_{k}}{x_{k}}\right)^{\alpha}\right)
$$

This is essentially the $\alpha$-logistic max-stable distribution in the literature, which is conventionally standardized to have 1-Fréchet marginals with scale parameter 1 (corresponding to $\left(\zeta_{1}^{\alpha}, \ldots, \zeta_{n}^{\alpha}\right)$ here). This is the only sub-Fréchet max-i.d. model which is also max-stable.

(ii) $L_{J_{*}}(t)=1 /(1+t)^{\theta}$, and (3.1) reads as

$$
\mathbb{P}\left(\zeta_{k} \leq x_{k}, k=1, \ldots, n\right)=\left(1+\sum_{k=1}^{n} \frac{\sigma_{k}}{x_{k}}\right)^{-\theta} \quad, \quad x_{k} \geq 0, k=1, \ldots, n
$$


Remark 3.5. The sub-Fréchet max-i.d. distributions can be extended immediately to max-i.d. random sup-measures, a topic which has raised some recent interest in the literature [14, 15]. Indeed, the law of the corresponding random sup-measures $\mathcal{M}$ is uniquely determined by its finite-dimensional distributions [22, Theorem 11.5], which essentially we have already computed in Proposition 3.1

Namely, we can define a family of max-i.d. random sup-measures on a generic measurable space $(E, \mathcal{E})$ equipped with a $\sigma$-finite measure $\mu$, in the form of

$$
\mathcal{M}(\cdot) \equiv \mathcal{M}_{\mu, \nu}(\cdot):=\bigvee_{\ell=1}^{\infty} J_{\ell} \mathcal{N}_{\ell}(\cdot),
$$

where $\boldsymbol{J} \equiv\left\{J_{\ell}\right\}_{\ell \in \mathbb{N}}$ a Poisson point process with mean measure $\nu$ as before and $\left\{\mathcal{N}_{\ell}\right\}_{\ell \in \mathbb{N}}$ are i.i.d. independently scattered 1-Fréchet random sup-measures on $(E, \mathcal{E})$ with control measure $\mu$ 21] , independent from $\boldsymbol{J}$. One can show that

$$
\mathcal{M} \stackrel{d}{=} J^{*} \mathcal{N}_{1}
$$

where $J^{*}$ is as before. Both cases in the previous example can be extended to the corresponding max-i.d. random sup-measures. Details are omitted.

Acknowledgements. We thank two anonymous referees for comments that helped improve the paper. SS's research was partially supported by the NSF FRG grant DMS-1462368. YW's research was partially supported by NSA grant H9823016-1-0322 and Army Research Laboratory grant W911NF-17-1-0006.

\section{REFERENCES}

[1] Berestycki, N. (2009). Recent progress in coalescent theory, volume 16 of Ensaios Matemáticos [Mathematical Surveys]. Sociedade Brasileira de Matemática, Rio de Janeiro.

[2] Davydov, Y., Molchanov, I., and Zuyev, S. (2008). Strictly stable distributions on convex cones. Electron. J. Probab., 13:no. 11, 259-321.

[3] de Haan, L. (1984). A spectral representation for max-stable processes. Ann. Probab., 12(4):1194-1204.

[4] Dombry, C. and Eyi-Minko, F. (2013). Regular conditional distributions of continuous max-infinitely divisible random fields. Electron. J. Probab, 18(7):1-21.

[5] Dombry, C. and Kabluchko, Z. (2018). Random tessellations associated with max-stable random fields. Bernoulli, 24(1):30-52.

[6] Dombry, C., Ribatet, M., and Stoev, S. (2017). Probabilities of concurrent extremes. Journal of the American Statistical Association, to appear.

[7] Dombry, C. and Zott, M. (2018). Multivariate records and hitting scenarios. Extremes, 21(2):343-361.

[8] Genest, C., Nešlehová, J. G., and Rivest, L.-P. (2018). The class of multivariate max-id copulas with $\ell_{1}$-norm symmetric exponent measure. Bernoulli, 24(4B):3751-3790.

[9] Gnedin, A. V. (2007). The chain records. Electron. J. Probab., 12:no. 26, 767786 .

[10] Goldie, C. M. and Resnick, S. (1989). Records in a partially ordered set. Ann. Probab., 17(2):678-699.

[11] Hashorva, E. and Hüsler, J. (2005). Multiple maxima in multivariate samples. Statist. Probab. Lett., 75(1):11-17. 
[12] Mai, J.-F. (2018). Extreme-value copulas associated with the expected scaled maximum of independent random variables. J. Multivariate Anal., 166:50-61.

[13] McNeil, A. J. and Nešlehová, J. (2009). Multivariate Archimedean copulas, $d$-monotone functions and $l_{1}$-norm symmetric distributions. Ann. Statist., 37(5B):3059-3097.

[14] Molchanov, I. and Strokorb, K. (2016). Max-stable random sup-measures with comonotonic tail dependence. Stochastic Process. Appl., 126(9):2835-2859.

[15] O'Brien, G. L., Torfs, P. J. J. F., and Vervaat, W. (1990). Stationary selfsimilar extremal processes. Probab. Theory Related Fields, 87(1):97-119.

[16] Pitman, J. (2003). Poisson-Kingman partitions. In Statistics and science: a Festschrift for Terry Speed, volume 40 of IMS Lecture Notes Monogr. Ser., pages 1-34. Inst. Math. Statist., Beachwood, OH.

[17] Pitman, J. (2006). Combinatorial stochastic processes, volume 1875 of Lecture Notes in Mathematics. Springer-Verlag, Berlin. Lectures from the 32nd Summer School on Probability Theory held in Saint-Flour, July 7-24, 2002, With a foreword by Jean Picard.

[18] Resnick, S. I. (1987). Extreme values, regular variation, and point processes, volume 4 of Applied Probability. A Series of the Applied Probability Trust. Springer-Verlag, New York.

[19] Samorodnitsky, G. and Taqqu, M. S. (1994). Stable non-Gaussian random processes. Stochastic Modeling. Chapman \& Hall, New York. Stochastic models with infinite variance.

[20] Stephenson, A. and Tawn, J. (2005). Exploiting occurrence times in likelihood inference for componentwise maxima. Biometrika, 92(1):213-227.

[21] Stoev, S. A. and Taqqu, M. S. (2005). Extremal stochastic integrals: a parallel between max-stable processes and $\alpha$-stable processes. Extremes, 8(4):237-266 (2006).

[22] Vervaat, W. (1997). Random upper semicontinuous functions and extremal processes. In Probability and lattices, volume 110 of CWI Tract, pages 1-56. Math. Centrum, Centrum Wisk. Inform., Amsterdam.

[23] Wadsworth, J. L. and Tawn, J. A. (2013). A new representation for multivariate tail probabilities. Bernoulli, 19(5B):2689-2714.

[24] Wang, Y. and Stoev, S. A. (2010). On the structure and representations of max-stable processes. Adv. in Appl. Probab., 42(3):855-877.

[25] Wang, Y. and Stoev, S. A. (2011). Conditional sampling for spectrally-discrete max-stable random fields. Adv. in Appl. Probab., 43(2):463-481.

Stilian Stoev, Department of Statistics, University of Michigan, Ann Arbor,, 439 W. Hall, 1085 S. University, Ann Arbor, MI 48109-1107, USA.

E-mail address: sstoev@umich.edu

Yizao Wang, Department of Mathematical Sciences, University of Cincinnati, 2815 Commons Way, Cincinnati, OH, 45221-0025, USA.

E-mail address: yizao.wang@uc.edu 